\title{
Modelling of Red Giant Stars: The state-of-the-art
}

\author{
Santi Cassisi ${ }^{1, \star}$ \\ ${ }^{1}$ INAF - Astronomical Observatory of Teramo, Via M. Maggini, sn, 64100 Teramo, Italy
}

\begin{abstract}
The seismic data obtained by the CoRoT and Kepler space missions have provided inferences of the global and structural properties of thousands of red giants. When compared with stellar model predictions, these inferences can significantly improve our understanding of stellar evolution. We present a brief review of the structure and evolution of red giant stars, devoting some emphasis on the major, still open problems.
\end{abstract}

\section{Introduction}

Red giants are cool, highly luminous stars, covering a rather wide domain in mass, age, chemical composition, and evolutionary stage. The three main classes of red giants: Red Giant Branch (RGB), Red Clump (RC) and Asymptotic Giant Branch (AGB) stars, provide a set of distance and star formation history diagnostics from photometric observations of resolved, old and intermediateage stellar populations.

The presence of an extended convective envelope can as in solar-like stars - stochastically excite p- and g-modes of oscillations that have indeed been detected with the CoRoT and Kepler space-based satellites. The use of these asteroseismological data allows robust constraints to be set on the mass and radius of such stars. As a consequence, since the data from the first CoRoT observational runs were analysed, and solar-like oscillations were detected in several thousands of red giant stars (mainly thanks to the Kepler space mission), it has become clear that the newly available observational constraints would allow novel approaches in the study of Galactic stellar populations [1, 2]. This can have a very important impact on our vision of the Galaxy. For instance, if the frequency at maximum power (the so called $v_{\max }$ ) of a red giant star, whose parallax is unknown, is derived from asteroseismic observations together with the so-called large frequency separation $(\Delta v)$, its radius can be estimated and so its distance derived: so oscillating red giants can become new distance indicators. In addition, a spectroscopic analysis of these stars, coupled with these new distance evaluations, could provide a new tool to measure the radial and vertical metallicity gradient in the Galaxy. Moreover, thanks to the estimate of the stellar mass through seismic constraints, it would be possible to characterise the age distributions and age-metallicity relations of the observed populations of giants [3].

Therefore, it appears evident that the impressive amount of seismic data for red giant stars is actually opening the way for 'ensemble seismology' of solar-like stars.

\footnotetext{
^e-mail: cassisi@oa-teramo.inaf.it
}

However, as a word of caution, one should recall that the seismic-based age estimates are inherently stellar model dependent, and hence affected by the still-present uncertainties in stellar evolution predictions. So, in order to improve the predictive capability of asteroseismic surveys, it appears mandatory to investigate the lingering uncertainties affecting stellar models for these stars, and to try to improve their accuracy and reliability.

We discuss the main evolutionary and structural properties of red giant stars separately discussing the case of RGB stars and RC ones, and outlining the main improvements that have been recently achieved in the modelling of these stars. We also briefly discuss some non-canonical physical processes - usually ignored in stellar model computations - that we need to properly account for in the next generation of stellar models in order to be able to properly interpret the various observational evidence.

\section{Red Giant Branch stars: their evolution up to the RGB Tip}

RGB stars are objects with masses lower than $\sim 2.2 M_{\odot}$ (the precise value depends on the initial chemical composition), which develop electron degenerate He-cores after the end of central H-burning, surrounded by a thick H-burning shell (thickness of the order of $0.1 M_{\odot}$ ) and a convective envelope. The envelope temperature gradient is to a large extent adiabatic, apart from the most external layers, where it becomes superadiabatic, and must be treated according to some prescribed convection theory (see Sect. 3.2).

At this stage, the convective envelope progressively deepens and the H-burning shell narrows down to a thickness of the order of $0.001 M_{\odot}{ }^{1}$, while the star crosses the Color-Magnitude diagram (CMD) towards redder colors (i.e. lower $T_{\text {eff }}$ and larger radius). There is a large body of literature devoted to the identification of what is (or

\footnotetext{
${ }^{1}$ This effect is due to the huge dependence on temperature of the $\mathrm{CNO}$ cycle that is controlling the H-burning process in the shell.
} 
what are, if more than one) the precise physical reason for the expansion of the star to red giant dimensions (a fact that comes out naturally from the integration of the stellar structure equations), but a general consensus has not been yet reached (see, e.g., [4] and references therein).

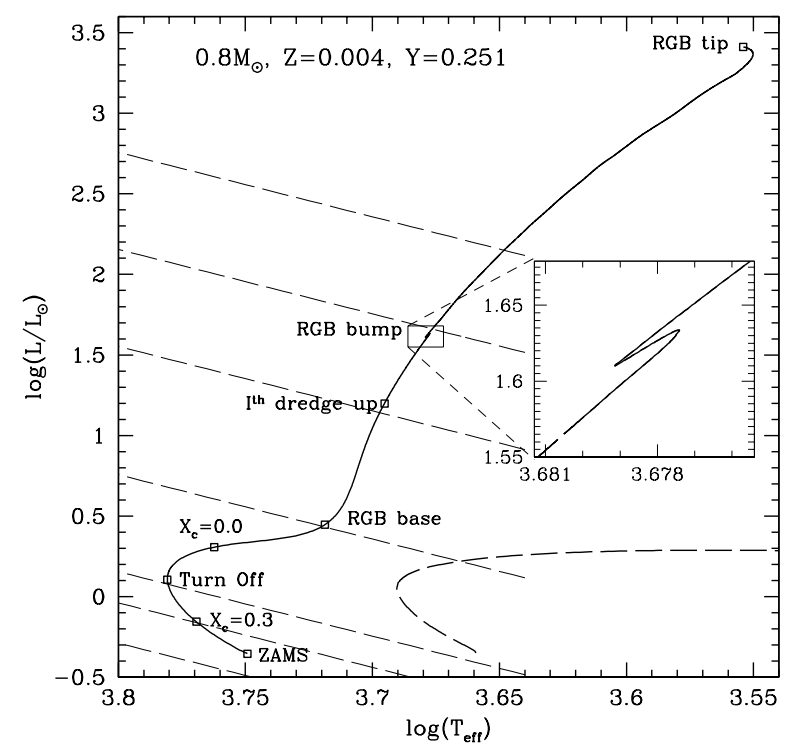

Figure 1: The evolution in the H-R diagram of a low-mass star from the ZAMS up to the RGB tip. The inset shows a enlargement of the RGB bump stage. The long dashed line corresponds to the track of the same mass, computed by setting the value of the mixing length parameter to zero. The thin dashed lines represent loci of constant radius for $\mathrm{R} / \mathrm{R}_{\odot}=0.6,0.8,1.0,2.0,5.0$, 10 and 20 , respectively, from bottom to top.

Due to the steady deepening of the convective region, the lower boundary of the convective envelope enters regions chemically processed (some produced $\mathrm{He}$ and the $\mathrm{C}$ and $\mathrm{N}$ abundances reaching their $\mathrm{CN}$-cycle equilibrium value) by the central H-burning phase; this material is mixed almost instantaneously throughout the convective envelope, thus altering the surface abundances. This phenomenon, called the first dredge up, causes an increase of the surface He abundance, and an increase of the N/C ratio with respect to the original values. Convection reaches its maximum extension (in mass) 'near' the base of the RGB, and from this moment on the lower convective boundary slowly recedes towards the surface, due to the steady growth of the He-core which accretes He produced by the H-burning shell.

The evolution along the RGB covers a relatively small range in $T_{\text {eff }}$ and a large range of surface luminosity, which increases with time. Due to the growth of the He core mass $\left(M_{\text {core }}^{H e}\right)$, the H-shell will encounter the sharp discontinuity of the H-profile left at the point of maximum extension of the convective envelope. The star reacts to the sudden increase of available fuel by lowering its surface luminosity and slowing down the evolutionary timescale, before starting again to increase its luminosity after the shell has moved past the discontinuity. This occurrence is recorded in the differential luminosity function (LF - number of stars in a given brightness interval as a function of the brightness itself) of old stellar populations as a local peak - the so-called RGB bump - in the differential LF.

When $M_{\text {core }}^{H e}$ reaches a size of about $0.50 M_{\odot}$ (the exact value depends weakly on the total mass of the star, being more sensitive to the initial chemical composition), Heignition occurs in the electron degenerate core, producing the so called He-flash which terminates the RGB phase by removing the electron degeneracy in the core, and drives the star onto its Zero Age Horizontal Branch (ZAHB) location, that marks the start of quiescent central He-burning plus shell $\mathrm{H}$-burning. Figure 1 shows the evolution of a $0.8 M_{\odot}(\mathrm{Z}=0.004, \mathrm{Y}=0.251)$ star from the Zero Age MS (ZAMS) up to the He-flash. The most relevant evolutionary stages are clearly indicated.

\section{On the modelling of RGB stars}

During these last decades, our knowledge of evolutionary and structural properties of stars is significantly improved. This result has been achieved as a consequence of our improved capability in understanding and describing the physical behavior of matter in the various thermal regimes characteristic of the various stellar mass ranges and evolutionary stages.

This notwithstanding, current generation of stellar models is still affected by significant and, usually, not negligible uncertainties. These uncertainties are related to our poor knowledge of some physical processes occurring in real stars such as, for instance, some nuclear reaction rates, the efficiency of mixing and diffusive processes. These drawbacks of stellar models have to be properly taken into account when comparing theory with observations in order to derive relevant information about the properties of both resolved and unresolved stellar populations, as well as when using stellar models for seismic purposes.

When facing with models for RGB stars, the main issues are those related with their age, effective temperature scale, the brightness of the RGB bump, and mass loss efficiency. In the following we will briefly review the accuracy and reliability of present generation of stellar models concerning these theoretical predictions.

\subsection{On the reliability of RGB stellar model age}

RGB timescales are of the order of a few percent of the main sequence lifetime, this has the important consequence that the uncertainty on the age of RGB stellar models is largely dominated by the uncertainty on the core $\mathrm{H}$-burning lifetime. When discussing this issue, we need to consider separately the case of low-mass stars, i.e. those that do not develop a convective core during the central $\mathrm{H}$ burning stage from those - the intermediate mass ones that burn $\mathrm{H}$ inside a convective core. The transition mass is around $1.1-1.2 M_{\odot}$ (the exact value depending on the chemical composition). For stars burning $\mathrm{H}$ in a radiative environment, the main sources of uncertainty in the core $\mathrm{H}$-burning lifetime are the nuclear reaction rates and the atomic diffusion efficiency. 

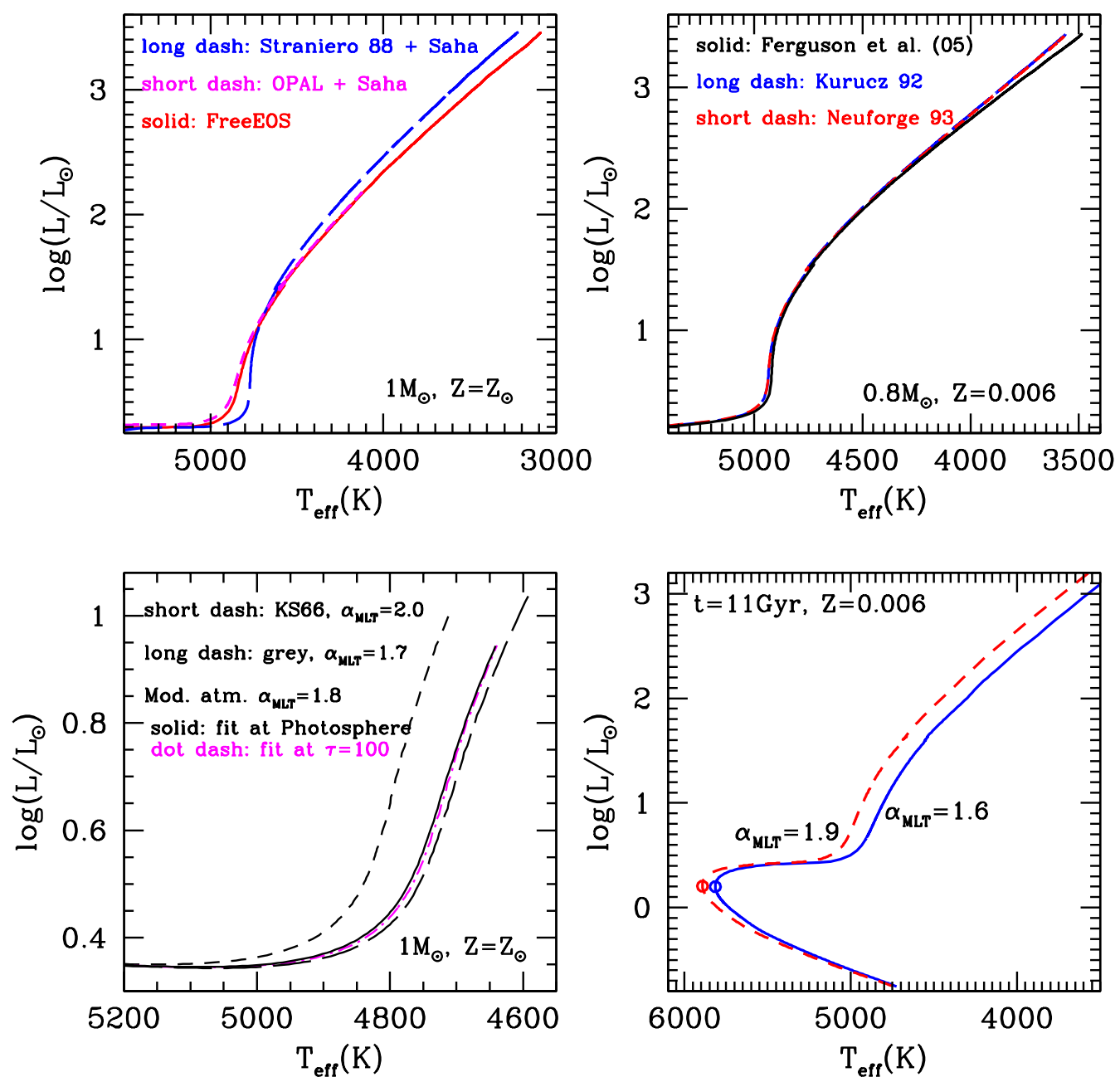

Figure 2: Upper left panel: the RGB portion of the evolutionary track of solar metallicity $1 \mathrm{M}_{\odot}$ models computed by adopting various EOSs. Upper right panel: the RGB loci of $0.8 \mathrm{M}_{\odot}$ model for a metallicity $\mathrm{Z}=0.006$, computed by using various prescriptions about the low-T radiative opacities. Lower left panel: the sub-giant branch and RGB portion of evolutionary tracks of various SSMs computed with various outer boundary conditions. For each model it has been also listed the corresponding value of the mixing length parameter. Lower right panel: two isochrones based on stellar models computed by using the same physical inputs but different values for $\alpha_{\mathrm{MLT}}$.

Concerning the nuclear reaction rates, one of the most important nuclear reaction in stellar astrophysics is the pcapture on ${ }^{14} \mathrm{~N}$ nuclei. At odds with the common idea that in low-mass stars the H-burning is always (fully) controlled by the nuclear reactions associated to the $p-p$ chain (whose efficiency is indeed very well known after the huge effort devoted to solve the solar neutrino problem), the nuclear reactions related to the $\mathrm{CNO}$-cycle can be actually important. In fact, near the end of the Main Sequence stage, due to the paucity of $\mathrm{H}$, the energy supplied by the H-burning becomes insufficient and the star reacts contracting its core in order to produce the requested energy via gravitation. As a consequence, both central temperature and density increase and, when the temperature reaches $\sim 15 \times 10^{6} \mathrm{~K}$, the H-burning process is controlled by the $C N O$ cycle, whose efficiency is critically dependent on the ${ }^{14} \mathrm{~N}(\mathrm{p}, \gamma){ }^{15} \mathrm{O}$ reaction rate, because this is the slowest reaction of the whole cycle.
In the past, its rate was uncertain, at least by a factor of 5 , because all available laboratory measurements were performed at energies well above the range of interest for astrophysical purposes. The LUNA experiment [5] has significantly improved the low energy measurements, obtaining an estimate which is about a factor of 2 lower than, for instance, the NACRE measurement. When considering an isochrone, the use of this updated reaction rate leads to a brighter and hotter MS turn-off (TO) for a fixed age (see $[6,7]$ and references therein): the use of these updated isochrones for Galactic globular cluster (GGC) age dating would translate in an increase of the cluster age of $\approx 0.9 \mathrm{Gyr}$ (that is about $10 \%$ of the age of old GGCs).

However, this result is mainly due to the change in the interior structure which influences the morphology of the evolutionary tracks, induced by the updated ${ }^{14} \mathrm{~N}(\mathrm{p}, \gamma)^{15} \mathrm{O}$ reaction rate. Indeed, when considering the impact on the evolutionary lifetime of individual stellar models, the age 

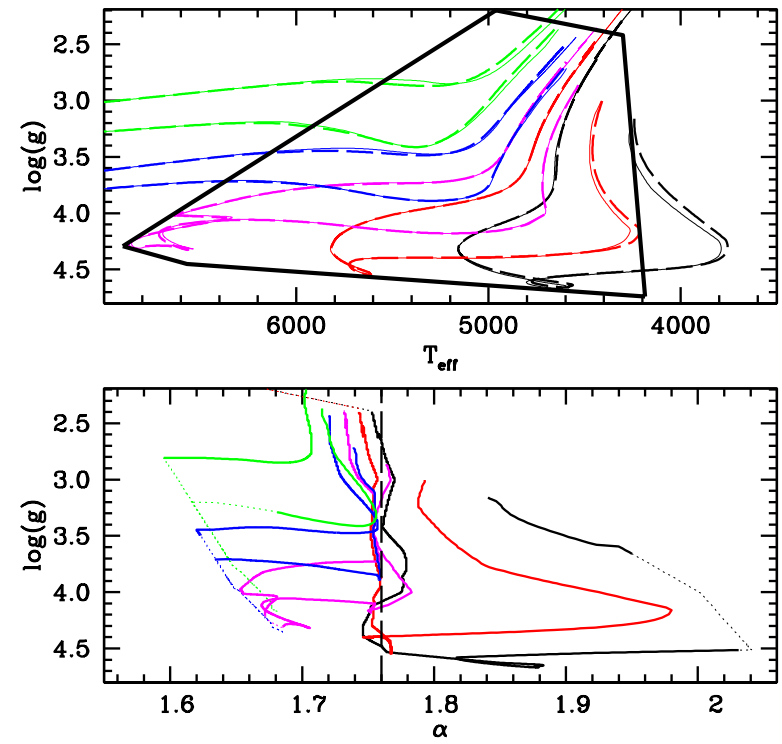

Figure 3: Upper panel: tracks for 0.7, 1.0, 1.4, 2.0, 3.0 $\mathrm{M}_{\odot}$ stellar models. The region enclosed by the thick black boundary is the $\mathrm{g}-T_{\text {eff }}$ range covered by the RHD simulations. Thin solid lines denote fully consistent calculations with the RHD calibrated variable mixing length and $\mathrm{T}(\tau)$ relationships. Lower panel: the evolution of the mixing length parameter along each track shown in the upper panel. The dotted portion of each sequence denotes the region where the $\alpha_{\text {MLT }}$ values have been extrapolated. Dashed lines in the upper panel display tracks calculated with a constant, solar-calibrated, mixing length equal to 1.76, and the calibrated $\mathrm{T}(\tau)$ relationship.

changes induced by the new rate amounts to (a negligible) $\approx 2 \%$. This means that the lingering uncertainties in the nuclear reaction rates for $\mathrm{H}$-burning processes have practically no impact when using individual stellar models for age dating in the framework of 'ensemble seismology'.

As far as it concerns the impact of atomic diffusion on evolutionary lifetimes, it has been shown that the inclusion of this process in stellar model computations for low-mass stars causes a decrease of the central H-burning lifetime of the order of $\approx 0.8-1$ Gyr (i.e. $\sim 10 \%$ of the whole core H-burning lifetime).

For stars developing a convective core during the central H-burning stage, several physical mechanisms could extend the size of convective cores beyond the Schwarzschild boundary, such as the overshooting of convective elements in the stable surroundings, and rotational mixings. These processes are still not satisfactorily understood by theory. Their combined effects are often modelled in the standard stellar model computations as a simple extension of the mixed convective core over a distance $\mathrm{d}_{\mathrm{ov}}=\lambda_{\mathrm{ov}} \times \mathrm{H}_{\mathrm{P}}$, where $\mathrm{H}_{\mathrm{P}}$ is the pressure scale height at the edge of the convective core, and $\lambda_{\mathrm{ov}}$ is a free parameter whose value has to be fixed. It is common to refer to $\mathrm{d}_{\mathrm{ov}}$ as the overshooting distance, even though overshooting may not be the only mechanism at work. This model is of course simplistic, but its simplicity reflects our current ignorance about the interface between convective and radiative regions. The value of the free parameter $\lambda_{\mathrm{ov}}$ can be fixed by trying to match some empirical constraints such as the CMD morphology around the MS TO in young and intermediate-age star clusters and/or the properties of well studied eclipsing binary systems (see, e.g. [8]).

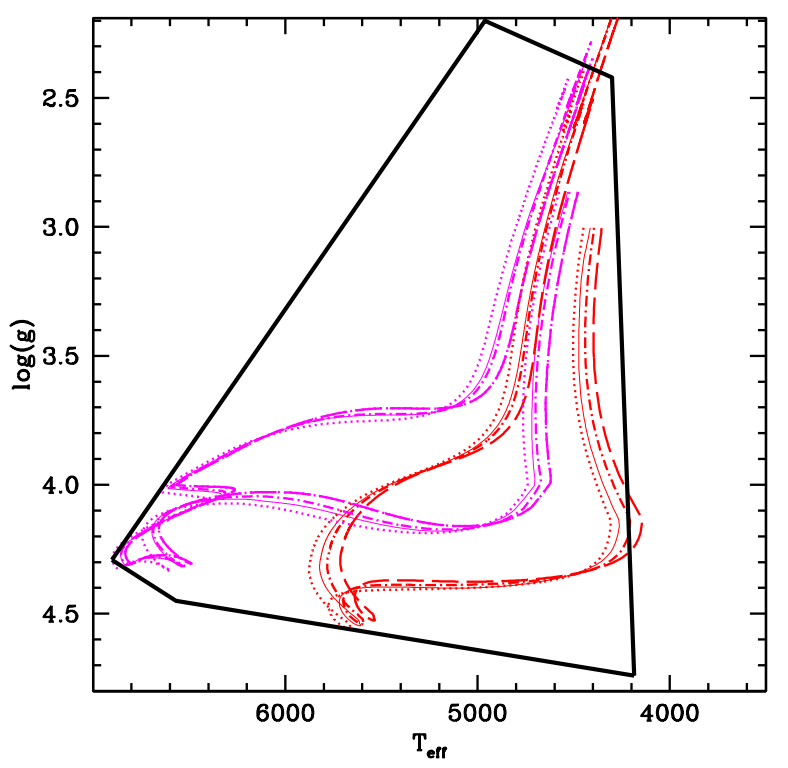

Figure 4: Lower panel: tracks for 1.0 and $1.4 \mathrm{M}_{\odot}$ models and various assumptions about the $\mathrm{T}(\tau)$ relation: thin solid lines correspond to fully consistent calculations with the RHD calibrated variable mixing length and $\mathrm{T}(\tau)$ relationships; dotted, dash-dotted and dashed lines display models calculated with constant $\alpha_{M L T}=1.76$ and the Eddington, KS66 and V81 T $(\tau)$, respectively.

When moving deeper inside the star, the pressure scale height steadily increases; this causes a large increase of the size of convective cores in stars whose Schwarzschild convective boundary is fast shrinking, (e.g., for masses below $\sim 1.5 \mathrm{M}_{\odot}$ ) if the overshooting efficiency is kept fixed at a constant fraction of $\mathrm{H}_{\mathrm{P}}$. This occurrence imposes that the value of $\lambda_{o v}$ has to be decreased to zero for stars with small convective cores. Due to the lack of any physicallygrounded prescription for how to deal with this issue, different assumptions about the trend of $\lambda_{O V}$ with the stellar mass can be adopted. The change of the isochrone morphology can be significant and different choices concerning the core overshoot efficiency in the critical mass range $1.1 \leq \mathrm{M} / \mathrm{M}_{\odot} \leq 1.5$ can mimic different isochrone ages. The obvious consequence is that the chosen trend of $\lambda_{O V}$ with mass, for masses with small convective cores, introduces an additional degree of freedom in stellar evolution models ([8]).

In these last years, a new approach has been envisaged to evaluate the extent of the convective core in central $\mathrm{H}$ burning stars. This method is based on the measurement of the effects on oscillation modes caused by the sharp variations in the mean molecular weight profile at the boundary of the convective core. Preliminary investigations ([9]) 
have clearly shown that this is a promising avenue. However, only recently seismic surveys of large sample of stars are really exploiting the great potential of asteroseismology to provide tight constrains on the real extension of the convective core (see, e.g., [10] and references therein). As a consequence important improvements in this context are expected in the near future.

\subsection{The $\mathrm{T}_{\text {eff }}$ scale of RGB stars}

The main parameters affecting the $\mathrm{T}_{\text {eff }}$ scale of RGB stars are: the equation of state (EOS), the low-temperature opacity, the efficiency of superadiabatic convection, the outer boundary conditions and the chemical abundances.

EOS: one of the most widely adopted EOS is the OPAL [11] one, whose range of validity does not cover the electron degenerate cores of RGB stars and their cooler, most external layers, below $5000 \mathrm{~K}$. As a consequence, RGB models computed with the OPAL EOS must employ some other EOS to cover the whole thermal stratification of RGB stars. However, there are some exceptions about this as in the case of the models computed by $[8,12,13]$. These sets of stellar models rely on the use of the FreeEOS provided by A. Irwin (2005) which consistently allows the computation of stellar models from the H-burning stage to the He-burning phase.

The upper left panel of fig. 2 shows the RGB of a $1 \mathrm{M}_{\odot}$ stellar structure computed adopting different assumptions about the EOS: the models based on the OPAL EOS and the FreeEOS are in very good agreement (this comparison is actually meaningful only for $\mathrm{T}_{\text {eff }}$ larger than about $4500 \mathrm{~K}$ for the reason discussed before); while there is a significant change in the RGB slope with respect to the model based on the EOS provided by [14] supplemented at the lower temperature by a Saha EOS. On average, there is a difference of about $100 \mathrm{~K}$ between RGB models based of the two different EOSs.

Low-T opacity: it has been shown by [15] that it is the low- $\mathrm{T}$ opacity which mainly determines the $\mathrm{T}_{\text {eff }}$ location of RGB models, while the high-T one - in particular opacity for temperatures around $10^{6} \mathrm{~K}$ - enters in the determination of the mass extension of the convective envelope. Present generations of stellar models employ mainly the low-T opacity calculations by [16] which are the most up-to-date computations suitable for stellar modelling, spanning a wide range of chemical compositions. The main improvement of this low-T opacity set with respect to older evaluations is in the treatment of molecular absorption and for a proper treatment of the presence of grains. Although significant improvements are still possible as a consequence of a better treatment of the different molecular opacity sources, we do not expect dramatic changes in the temperature regime where the contribution of atoms and molecules dominate, whilst large variations can be foreseen in the regime $(\mathrm{T}<2000 \mathrm{~K})$ where grains dominates the interaction between radiation and matter.

Upper right panel of fig. 2 shows a comparison between RGB models computed by adopting the same physical inputs but different low-T opacity evaluations: for effective temperature larger than $\sim 4000 \mathrm{~K}$, the $\mathrm{T}_{\text {eff }}$ scale of the models computed by using the (nowadays obsolete) opacities provided by [17] and [18] is in very good agreement with that corresponding to the model computed by adopting the [16]'s opacities. However, as soon as the RGB $\mathrm{T}_{\text {eff }}$ goes below this limit (i.e., when the models approach the RGB tip and/or their metallicity is increased), the opacities by [16] produce progressively cooler models (differences reaching values of the order of $100 \mathrm{~K}$ or more), due to the increasing effect of the molecules - in particular $\mathrm{H}_{2} \mathrm{O}$ and $\mathrm{TiO}$ ones - which contribute substantially to the (increase of the) opacity in this temperature range.

Outer boundary conditions: the procedure commonly adopted for fixing the boundary conditions at the bottom of the atmospheric layers, relies on the integration of the atmosphere by using a functional (semi-empirical or theoretical) $(\mathrm{T}(\tau))$ relation between the temperature and the optical depth $\tau$ ). The effect of using boundary conditions from model atmospheres on RGB stellar models has been extensively investigated by [19]. In lower left panel of fig. 2 it is shown the effects of different $\mathrm{T}(\tau)$ relations, namely, the Krishna-Swamy (KS66) solar T( $\tau$ ) [20] relationship and the Eddington (grey) one, on a $1 \mathrm{M}_{\odot}$ solar metallicity model computed by using the same physical inputs and a solar calibrated mixing length parameter. By construction all tracks overlap at the present solar luminosity and effective temperature. On the RGB the track based on the KS66 atmosphere is $\sim 100 \mathrm{~K}$ hotter than the tracks based on the model atmosphere stratification (in the specific case the MARCS models have been used - see [19] for more details) and the Eddington $\mathrm{T}(\tau)$ relationship. It is also important to note that the two tracks obtained matching the MARCS atmospheres to the interior structure at different optical depths: $\tau=1$ and 100, respectively, do overlap from the Zero Age MS to the RGB. This occurrence is also valid at lower metallicities, and is a proof that the stellar model $\mathrm{T}_{\text {eff }}$ scale does not depend on the chosen fitting point until a value of $\tau$ larger or equal to unity is chosen.

When comparing more metal-poor, low-mass stellar models - based on solar-calibrated $\alpha_{\text {MLT }}$-, the difference in the $\mathrm{T}_{\text {eff }}$ scale between models based on both types of grey atmospheres and detailed non-grey models appears negligible. It is worth noting that the comparison between theory and observations for the effective temperature scale of approximately solar metallicity RGB stars both in the field and in star clusters, seems to support the $\mathrm{T}_{\text {eff }}$ scale of stellar models based on the KS66 T $(\tau)$ relation.

Before closing this discussion, we wish to remark that, even if the use of boundary conditions provided by model atmospheres is, in principle, more rigorous, one has also to bear in mind that the convection treatment in the adopted model atmospheres is usually not the same as in the underlying stellar models (i.e., a different mixing length formalism and a different value for the scale height of the convective motion are used).

The treatment of superadiabatic convection: the temperature gradient throughout the bulk of the convective en- 
velope of stellar models can be approximated by the adiabatic value. However, in the outer layers close to the stellar surface the convective gradient becomes strongly superadiabatic. To determine the actual value of the gradient in these outer layers the Mixing Length Theory (MLT, [21]) is almost universally used. The MLT contains four free parameters, whose values affect the predicted $\mathrm{T}_{\text {eff }}$ of the stellar models. Three parameters are fixed a priori ${ }^{2}$ (and define what it is denoted as the MLT flavour), and the only one left to be calibrated is $\alpha_{\mathrm{MLT}}$, the ratio of the mixing length to the local pressure scale height $\mathrm{H}_{\mathrm{P}}$, that provides the scale length of the convective motions. As a general rule, an increase of $\alpha_{\mathrm{MLT}}$ corresponds to an increase of the convective transport efficiency and an increase of the stellar model $\mathrm{T}_{\text {eff }}$.

In stellar evolution calculations the value of $\alpha_{\mathrm{MLT}}$ is usually calibrated by reproducing the radius of the Sun at the solar age with a Solar Standard Model (SSM). This solar calibrated $\alpha_{\mathrm{MLT}}$ is then kept fixed in all evolutionary calculations of stars of different masses and chemical compositions. The exact numerical value of $\alpha_{\mathrm{MLT}}$ varies amongst calculations by different authors because variations of input physics and choices of the outer boundary conditions affect the predicted model $\mathrm{T}_{\text {eff }}$ values, hence require different $\alpha_{\text {MLT }}$ values to match the Sun.

It is clear that, even if a physics input employed in the stellar model computations is not accurate, it is possible to mask this shortcoming - at least from the point of view of the predicted $\mathrm{T}_{\text {eff }}$ - by simply recalibrating $\alpha_{\mathrm{MLT}}$ on the Sun. This guarantees that the models always predict correctly the $\mathrm{T}_{\text {eff }}$ of at least solar-type stars. However, since the extension of the superadiabatic layers is larger in RGB stars, theoretical RGB models are much more sensitive to $\alpha_{\text {MLT }}$ than MS ones. Therefore it is not safe to assume a priori that the solar calibrated value of $\alpha_{\mathrm{MLT}}$ is also adequate for RGB stars of various metallicities.

A source of concern about a priori assumption of a solar $\alpha_{\mathrm{MLT}}$ for RGB computations comes from the fact that recent models from various authors, all using a suitably calibrated solar value of $\alpha_{\mathrm{MLT}}$, do not show the same RGB temperatures. A comparison - performed by [23] - of independent sets of RGB stellar models computed with the same initial chemical composition and solar calibrated values of $\alpha_{\text {MLT }}$ shows that these models can predict a different $\mathrm{T}_{\text {eff }}$ scale for the RGB: a realistic estimate of the current uncertainty on this $\mathrm{T}_{\text {eff }}$ scale is of the order of $200-300 \mathrm{~K}$. The reason for this discrepancy must be due to some difference in the input physics which is not compensated by the solar calibration of $\alpha_{\mathrm{MLT}}$. This occurrence clearly points out the fact that one cannot expect the same RGB $\mathrm{T}_{\text {eff }}$ scale from solar calibrated models that do not employ exactly the same input physics.

\footnotetext{
${ }^{2}$ We refer to [22] for a detailed analysis of the impact of choosing different values for these free parameters on stellar model computations. However, as a general rule, these authors have shown that sets of models - based on distinct MLT flavours but the same physical inputs - provide consistent results with only minor differences, once the mixing length has been properly calibrated on the Sun. The same sets of models are also able to reproduce the $\mathrm{T}_{\text {eff }}$ of RGB stars in GGCs.
}

The MLT formalism provides only a very simplified description of convection, and there have been several attempts to introduce non-locality in the MLT (see, e.g., [24], and references therein). These 'refinements' are often complex and introduce additional free parameters to be calibrated. The alternative model by [25] includes a spectrum of eddy sizes (rather than the one-sized convective cells of the MLT) and fixes the scale length of the convective motions to the distance to the closest convective boundary. [26] have presented a novel non-local and time-dependent model based on the solution of the NavierStokes equations for an incompressible perfect fluid - the so called scale-free convection theory (SFC) -, that does not contain free parameter. A few stellar models based on this SFC have been recently presented by [27]: these preliminary computations seem to shown that, at least for MS stars, the SFC theory yields results very similar to those derived from a solar calibrated MLT regarding the extension of the convective zones, temperature gradients, and energy fluxes, whereas for RGB stars the differences between sets of models based on the two convection theories are significant. Nevertheless, we note that recently the physical basis for this approach for the treatment of the superadiabatic convection has been strongly questioned by [28].

An alternative approach to model the superadiabatic layers of convective envelopes is based on the computation of realistic multidimensional radiation hydrodynamics (RHD) simulations of atmospheres and convective envelopes - where convection emerges from first principles - that cover the range of effective temperatures, surface gravities, and chemical compositions typical of stars with convective outer regions. These simulations have reached nowadays a high level of sophistication ([29]) and for ease of implementation in stellar evolution codes, their results can be used to provide an 'effective hydro-calibration' of $\alpha_{\mathrm{MLT}}$, even though RHD simulations do not confirm the basic MLT picture of columns of convective cells. In this context, [30] produced a grid of convective atmosphere/envelope 3D RHD simulations for the solar chemical composition. The same grid of 3D RHD simulations have been matched ([31]) to 1D hydrostatic equilibrium, spherically symmetric envelope models to calibrate $\alpha_{\text {MLT }}$ as function of gravity and effective temperature ${ }^{3}$

Moreover, the same RHD simulations have been employed by [32] to calculate $\mathrm{g}$ - and $\mathrm{T}_{\mathrm{eff}}$-dependent temperature relations as a function of the $\tau$ Rosseland optical depth. The availability ([31]) of a numerical routine to calculate a g- and $\mathrm{T}_{\text {eff-dependent RHD-calibrated } \alpha_{\mathrm{MLT}} \text { and }}$ $\mathrm{T}(\tau)$ relations and Rosseland opacities consistent with the opacities used in the RHD simulations, enables stellar evolution calculations where boundary conditions, superadiabatic temperature gradient and opacities of the convective envelope are consistent with the RHD simulations ${ }^{4}$.

\footnotetext{
${ }^{3}$ These RHD simulations provide a value for the $\alpha_{\text {MLT }}$ for the Sun equal to $1.76 \pm 0.03$, not very different from the values obtained with the calibration of SSMs.

${ }^{4} \mathrm{We}$ remark that it is particularly important to use both the RHDcalibrated $\alpha_{\text {MLT }}$ and T $(\tau)$ relations, because the $T_{\text {eff }}$ scale of the stellar models depends on both these inputs as discussed by [23] and [19].
} 

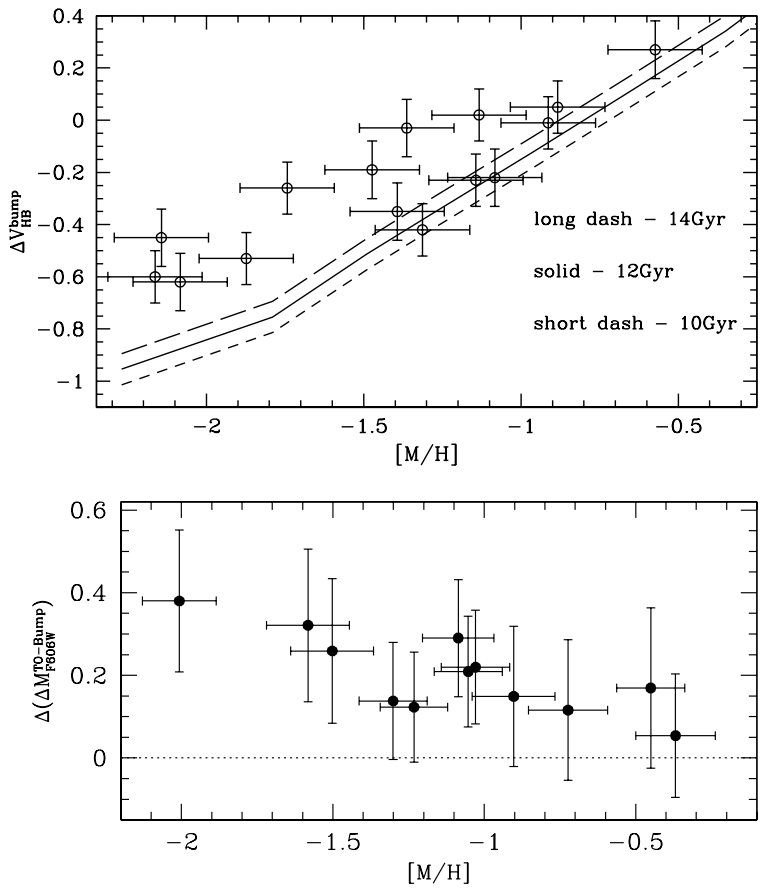

Figure 5: Upper panel: comparison between estimates of the brightness difference between the RGB bump and the ZAHB for a sample of Galactic globular clusters, and BaSTI theoretical prescriptions - for the labeled assumptions about the cluster age - as a function of the global metallicity. Lower panel: difference between the values of $\Delta \mathrm{M}_{\mathrm{F} 606 \mathrm{~W}_{\mathrm{TO}}}$ Bump predicted by models for each individual GC - once its age has been obtained from the absolute TO brightness -, and empirical values, as a function of $[\mathrm{M} / \mathrm{H}]$.

[33] have been the first to present stellar evolution calculations where this 3D RHD-calibration of $\alpha_{\mathrm{MLT}}$ is self-consistently included in a stellar evolution code. Figure 3 shows some results from the quoted investigation: a comparison between fully consistent model calculations with the RHD calibrated variable $\alpha_{\text {MLT }}$ and T $(\tau)$ relationships, and models based on a constant, solar calibrated $\alpha_{\text {MLT }}=1.76$ and the same $\mathrm{T}(\tau)$ relationship. From the point of view of the predicted $\mathrm{T}_{\text {eff }}$ scale, models calculated with constant RHD calibrated solar mixing length $\alpha_{\mathrm{MLT}}=1.76$ are very similar to, and often quite indistinguishable from, the models with variable $\alpha_{\mathrm{MLT}}$, maximum differences being at most $\sim 30-50 \mathrm{~K}$. Indeed, the same analysis has provided a plain evidence that the choice of RHD-calibrated $\mathrm{T}(\tau)$ relation is more relevant than the use of a variable $\alpha_{\mathrm{MLT}}$, in setting the effective temperature scale of the models as shown in Fig. 4: here it is shown the comparison between models based on the RHD-calibrated $\mathrm{T}(\tau)$ relation and the $\mathrm{T}(\tau)$ relationships by Eddington, KS66 and [34] (V81).

\subsection{The Red Giant Branch luminosity function}

The RGB luminosity function (LF) of GGCs is an important tool to test the chemical stratification inside the stellar envelopes (see [35] and references therein). The RGB LF is a simple straight line on a magnitude- $\log (N)$ plane, except in a very narrow magnitude interval corresponding to the RGB bump. This linearity is a direct consequence of the He-core mass - luminosity relation for RGB stars ([35]). The slope of this line allows a major test of the evolutionary rate along the RGB, that is virtually independent of the isochrone age or metallicity. Comparisons between theoretical LFs and empirical ones for GGCs have shown a remarkable good agreement: this represents a plain evidence of the fact that theoretical predictions about the evolutionary rate along RGB are quite reliable.

As previously discussed, the brightness of the RGB bump is related to the location of the $\mathrm{H}$-abundance discontinuity left over by outer convection during the first dredge $u p$, in the sense that the deeper the chemical discontinuity is located, the fainter is the bump luminosity. Since any physical inputs and/or numerical assumption adopted in the computations which affects the maximum extension of the convective envelope at the first dredge up, strongly affects the bump brightness, a comparison between the predicted RGB bump luminosity and observations in star clusters allows a direct check of how well theoretical models for RGB stars predict the extension of convective regions in the stellar envelope and, then provide a plain evidence of the reliability of the adopted evolutionary framework ([36]).

However, when comparing theory with observations one needs a preliminary estimate of both the cluster metallicity and distance. Current uncertainty in the GGC metallicity scale strongly reduces our capability to constrain the plausibility of the theoretical framework, and for such reason, it has became a common procedure to use simultaneously all available metallicity scales (see [37] for a discussion on this issue). An additional critical issue is related to the knowledge of the cluster distance, whose indetermination could strongly hamper the possibility of a meaningful comparison between theory and observations. In order to overcome this problem, as early suggested by [38], the observed V-magnitude (or filters similar to Johnson V) difference between the RGB bump and the HB at the RR Lyrae instability strip $\left(\Delta V_{\mathrm{HB}}^{\mathrm{Bump}}=\mathrm{V}_{\mathrm{HB}}-\mathrm{V}_{\text {bump }}\right)$ is usually adopted in order to test the theoretical predictions for the bump brightness. This quantity presents several advantages from the observational point of view and it is empirically well-defined because it does not depend on a previous knowledge of the cluster distance and reddening. However, on the theoretical side, one should keep in mind that such comparison requires the use of a theoretical prediction about the Horizontal Branch brightness which is a parameter still affected by some uncertainty. Nevertheless, empirical estimates about the $\Delta \mathrm{V}_{\mathrm{HB}}^{\text {Bump }}$ parameter have been extensively compared with theoretical predictions (see [39] and references therein).

Upper panel of fig. 5 shows a comparison between recent measurements of this parameter for a sample of GGCs and theoretical predictions, and discloses a discrepancy at the level of $\sim 0.20 \mathrm{mag}$, or possibly larger - , for GCs with $[\mathrm{M} / \mathrm{H}]$ below $\sim-1.5$ : the predicted $\Delta \mathrm{V}_{\mathrm{HB}}^{\text {Bump }}$ being larger than observed. We cannot discriminate whether this 
is due to too bright theoretial bump luminosities, or underluminous HB models (or a combination of both effects). Due to the strong dependence of $\Delta \mathrm{V}_{\mathrm{HB}}^{\mathrm{Bump}}$ parameter on the metallicity, a more quantitative evaluation of the 'real' discrepancy between theory and observation strongly depends on the adopted metallicity scale.

A complementary avenue is offered by the magnitude difference between the TO and RGB bump, $\Delta \mathrm{V}_{\mathrm{TO}}^{\text {Bump }}=\mathrm{V}_{\mathrm{TO}}-\mathrm{V}_{\text {bump }}$, which bypasses the HB. Lower panel of fig. 5 shows a comparison between this quantity as measured for a sample of GGCs observed in the F606W filter of the Advanced Camera for Surveys on board of HST and the similar theoretical quantity as estimated once the age of each GC has been accurately derived via the isochrone fitting to the absolute MS TO magnitude obtained using a MS-fitting distance scale (see [40], for a more detailed discussion on the adopted method). This plot shows clearly that the expected $\Delta \mathrm{M}_{\mathrm{F} 606 \mathrm{~W}_{\mathrm{TO}}}$ Bump values are systematically larger than observed: the mean difference being of the order of $0.20 \mathrm{mag}$. Given that the observed TO magnitude is by definition matched by the theoretical isochrones to determine the TO age, this discrepancy implies that the theoretical RGB bump luminosity is too bright, e.g., RGB models predict a too shallow maximum depth of the envelope at the first dredge up.

On the basis of the most recent updates in the input physics, it does not appear realistic to modify the maximum depth of the convective envelope at the first dredge up by reasonable changes in the adopted physics (i.e., in the radiative opacity). The most viable solution of this discrepancy implies some amount of convective overshoot, beyond the Schwarzschild convective boundary, by about $0.25 \mathrm{H}_{\mathrm{P}}{ }^{5}$. The inclusion of atomic diffusion can also help in reducing the discrepancy but it can not completely solve the problem.

New, independent, insights concerning the location of the chemical discontinuity in the envelope of RGB stars can be provided by an accurate analysis of the g-modes in oscillating giants. In fact, recently [41] have investigated the possibility to use the perturbations (also called glitches) of the frequencies of high-radial-order g-modes from their asymptotic value, due to sharp variations in the buoyancy frequency induced by local changes in the chemical composition. Although no robust observational results on these buoyancy frequency glitches have been presented so far, one can predict that - once observed - this kind of seismic effects can represent a valuable tool for investigating the inner chemical stratification of cool stars.

In passing, we also note that the RGB LF bump provides other important constraints besides brightness for checking the accuracy of RGB models. More in detail, both the shape and the location of the bump along the RGB LF can be used for investigating on how 'steep' is the Hdiscontinuity left over by envelope convection at the first dredge up. So these features appear, potentially, a useful tool for investigating on the efficiency of non-canonical mixing at the border of the convective envelope ([42]) able

\footnotetext{
${ }^{5}$ We note that the RGB bump brightness changes at the rate of $0.8 \mathrm{mag} / \mathrm{H}_{\mathrm{P}}$.
}

to partially smooth the chemical discontinuity. In addition, since the evolutionary rate along the RGB is strongly affected by any change in the chemical profile, it is clear that star counts in the bump region can provide reliable information about the size of the jump in the $\mathrm{H}$ profile left over by envelope convection after the first dredge up ([37]).

\subsection{Mass-loss efficiency along the RGB stage}

A major issue in stellar evolution theory is the treatment of the mass loss (ML) during the advanced evolutionary stages. In fact, reliable empirical ML determinations, as well as a comprehensive physical description of the involved processes are so far still lacking; in particular, there is a lack of any empirical law directly calibrated on Population II giants. From a theoretical point of view, our knowledge of the ML timescales, driving mechanisms, dependence on stellar parameters and metallicity is also very poor. The consequence is that there is little theoretical or observational guidance on how to incorporate ML into stellar model computations.

As for the ML efficiency along the RGB, mass loss rates are customarily parametrized in stellar model calculations by means of simple relations like the Reimers formula ([43]). A few other formulae, which are variants of the Reimers one, have been proposed but there is no a priori reason for choosing one amongst the different alternatives. These prescriptions are essentially scaling relations between mass loss rates and global stellar parameters like, e.g., surface bolometric luminosity and gravity, effective temperature and/or radius. The zero point of these scaling relations is typically set by a free parameter $(\eta)$ that needs to be calibrated.

The last decade has witnessed a growing amount of empirical data concerning ML estimates for Pop. II red giants, based on a more direct approach as the detection of outflow motions in the outer regions of the atmospheres (see, e.g., [44]), or the detection of the circumstellar envelopes at larger distances from the stars (see, e.g., [45]). These empirical analyses suggest that the 'actual' ML law should be significantly different (flatter) than the Reimers formula, that seems to be ruled out at the $3 \sigma$ level. In addition, the RGB ML phenomenon seems to be not a continuous process but an episodic phenomenon, whose efficiency does not appear to be strongly correlated with the metallicity. Recently, [46, 47] applied an alternative approach to estimate the mass lost by RGB stars in the GGC 47 Tuc. These authors determined the rate of diffusion of stars through the cluster core, using a sample of young white dwarfs, and compared the radial distribution of upper MS, RGB and HB stars. As a result, they found that the radial distributions of the various classes of stars are nearly identical, showing that there has been very little time for the young WDs to have diffused through the cluster since their progenitors lost mass. Based on these dynamical consideration, [47] estimated that up to twothirds of the $\sim 0.4 \mathrm{M}_{\odot}$ that 47 Tuc stars are expected to lose between the end of the MS and the beginning of the WD sequence, is shed shortly before the start of the WD cooling, and a typical HB stellar mass of the order of $\sim 0.65 \mathrm{M}_{\odot}$ 
(that corresponds to a mass loss of about $0.25 \mathrm{M}_{\odot}$ along the RGB) is excluded by comparing the radial distribution of HB stars and MS stars.

Needless to say that this result would severely challenge the common idea about the efficiency of mass loss during the RGB stage. Actually, the need for a significant ML during the RGB in order to reproduce the observed cluster HB morphology has been shown by [48]: a detailed comparison between synthetic HB models and data shows that the observed morphology can be reproduced only by accounting for an average mass lost during the $\mathrm{RGB}$ equal to $\Delta \mathrm{M} \approx 0.23 \mathrm{M}_{\odot}$ and an almost negligible Gaussian spread of $0.005 \mathrm{M}_{\odot}$ around this value. When taking into account the possible sources of uncertainty related to the cluster properties such as reddening, chemical composition (also in the context of the multiple population phenomenon) and age, [48] found that the average mass lost during the RGB ranges from a minimum value of $\sim 0.17 \mathrm{M}_{\odot}$ (for a $\mathrm{GC}$ age of $12.5 \mathrm{Gyr}$ ) to a maximum value of $\sim 0.21 \mathrm{M}_{\odot}$ (for a GC age of $10.5 \mathrm{Gyr}$ ). This analysis suggests the existence of a significant discrepancy between the information coming from cluster dynamics and CMD modelling of the HB. A comparison between the results from these two techniques applied to other GCs is required, to gain more insights about the origin of this apparent disagreement.

An independent approach for measuring the ML efficiency in RGB stars has been adopted by [49]: thanks to the Kepler detection of solar-like oscillations in G-K giants in the old open clusters NGC 6791 and 6819, they posed a constraint on the integrated RGB mass-loss efficiency by comparing the average mass of RC stars with that of stars in the faint portion of RGB. As a results they found that very efficient RGB ML can be excluded, and the available data are consistent with ML rates described with a Reimers $\eta$ parameter smaller than $\sim 0.35$. These results are interesting, and the seismic method appears a quite viable way to estimate ML efficiency. However, since stellar masses are determined by combining the available semiotics parameters $v_{\text {max }}$ and $\Delta v$ with other data (as photometry and distance determinations), more accurate and reliable stellar masses determinations are expected from additional constraints from individual pulsation mode frequencies, spectroscopic $\mathrm{T}_{\text {eff }}$ determinations and accurate tests of the adopted $v_{\text {max }}$ and $\Delta v$ scaling relations.

Before closing this section, we wish to emphasise that an accurate determination of the RGB ML efficiency is a crucial issue also in the context of using stellar models and seismic measurements for Galactic archaelogy ([2]). Indeed, one has to bear in mind that the computation of ages for red giant stars (both RGB stars and RC ones) heavily relies on the knowledge of their masses. In the context of asteroseismologic surveys, the mass of a star is essentially derived by using scaling relations based on the observed $\Delta v$ and $v_{\text {max }}$, and thus the mass is rather independent of the adopted set of stellar models. The same, however, does not hold for ages. Although the mass of a red giant is a useful proxy for age, it is important to distinguish between the initial mass, which sets the evolutionary lifetime of a star,

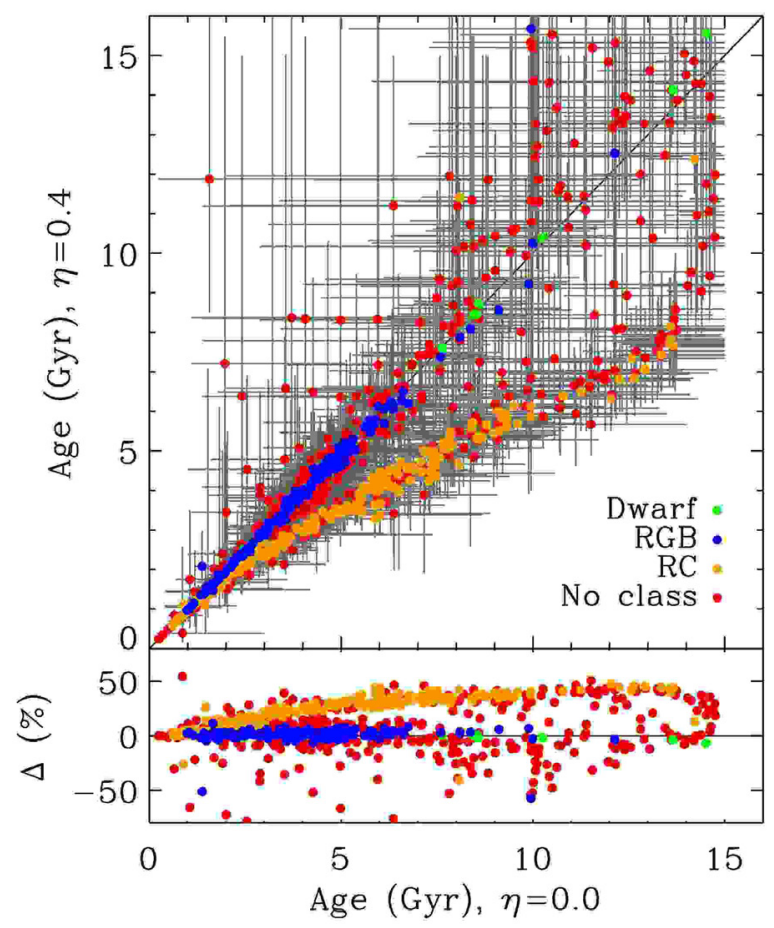

Figure 6: Comparison between ages obtained by alternatively using stellar models accounting for mass-loss (with the parameter $\eta$ equal to 0.4 ) and not accounting for ML.

[Reproduced with permission from [3], published by Oxford University Press on behalf of The Royal Astronomical Society. All rights reserved. Available online at http://dx.doi.org/10.1093/mnras/stv2320. This figure is not covered by the Open-Access licence of this publication. For permissions contact Journals.permissions@OUP.com]

and the present-day (i.e., the actual) stellar mass, which is indeed the quantity derived from seismology. Thus, when inferring an age using the actual stellar mass, the value derived will depend on the past history of the star, whether or not significant ML has occurred during its evolution. This issue has been investigated in detail by [3].

Figure 6 shows the comparison of between ages derived with and without mass-loss. Ages of dwarf stars are obviously unaffected by mass-loss, and the same conclusion holds for RGB stars. ${ }^{6}$ The effect of ML increases when moving to lower gravities, and it is quite dramatic for stars in the central He-burning stage. Theoretical isochrones including ML return younger ages than those without ML; this can be easily understood since a given mass - seismically inferred - will correspond to a higher initial mass in case of ML, whose evolutionary lifetimes are shorter.

\subsection{Additional physical processes}

Standard models for red giant stars are non-rotating, spherically symmetric and usually include only convection as

\footnotetext{
${ }^{6}$ The distinction between RGB and RC stars is based on the average spacing between mixed modes ([50]); hence a robust identification of RGB stars is possible for $\log (g) \geq 2$.6, i.e. on the lower part of the RGB, where ML turns out to be of little importance in the Reimers formulation. This explains the weak dependence of RGB ages on mass-loss.
} 
element transport mechanism. These models predict that, during the first dredge up, the surface chemical composition of low-mass stars is modified due to dredge up into the convective envelope of $\mathrm{H}$-burning processed material. After this mixing episode, canonical models do not predict any further mixing episode along the RGB. Several spectroscopic observations of metal poor Galactic halo stars provide however compelling evidence of an additional mixing process occurring when RGB stars reach the luminosity of the RGB bump: a sudden drop of the isotopic ratio ${ }^{12} \mathrm{C} /{ }^{13} \mathrm{C}$, a decrease of the $\mathrm{Li}$ and $\mathrm{C}$ abundances and an increase of the $\mathrm{N}$ abundance is clearly shown by the empirical data. This 'non-canonical' mixing seems to be a universal process as it affects $\sim 95 \%$ of low-mass stars, regardless of whether they populate the halo field or clusters (see [51] and references therein). The mechanism that has been proposed as a possible source for this mixing episode should be associated with the molecular weight inversion due to the ${ }^{3} \mathrm{He}$ burning through the ${ }^{3} \mathrm{He}\left({ }^{3} \mathrm{He}, 2 \mathrm{p}\right){ }^{4} \mathrm{He}$ nuclear reaction, in the outer wing of the H-burning shell, and the associated thermohaline mixing.

In low-mass stars, the main burning mechanism during the MS is the $p$ - $p$ chain, that due to its weak dependence on temperature, is efficient also in stellar layers quite far from the star centre. As a consequence, ${ }^{3} \mathrm{He}$ accumulates in a broad zone outside the main energy production region. During the first dredge up this ${ }^{3} \mathrm{He}$ is mixed within the convective envelope, with the consequence that during the following RGB evolution, the layers above the $\mathrm{H}$ discontinuity left over by the receding convective envelope at its maximum extension will have a uniform ${ }^{3} \mathrm{He}$, larger than the initial one. When the shell advances towards the surface during the RGB evolution, in the outer wing above the point of maximum burning efficiency, there is a narrow region where ${ }^{3} \mathrm{He}$ is burnt through the reaction ${ }^{3} \mathrm{He}\left({ }^{3} \mathrm{He}, 2 \mathrm{p}\right){ }^{4} \mathrm{He}$. This nuclear reaction is unusual in the sense that 2 nuclei transform into 3 and the mean mass per nucleus decreases from 3 to 2 . Because the molecular weight is the mean mass per nucleus, this leads to a small local decrease in the $\mu$ gradient. As long as the H-burning shell moves through layers below the H-discontinuity - i.e. the star evolves before the RGB bump - this local negative $\mu$ gradient is negligible because the shell is moving in a region with a much larger positive gradient, due to the He profile left over at the end of the MS. However, when the H-burning shell enters the region of uniform chemical profile beyond the $\mathrm{H}$-discontinuity, the local inversion, i.e. negative gradient, in the $\mu$ profile, of the order of one part in $10^{4}$, becomes much more important. This situation corresponds to the conditions for thermohaline mixing (see [52] and references therein). This mixing is usually included in stellar evolution codes as a diffusive process that works in the direction to erase the molecular weight inversion, with diffusion coefficient given by [53]

$$
\mathrm{D}_{\text {th }}=\mathrm{C}_{\text {th }} \frac{K}{\mathrm{c}_{\mathrm{p}} \rho}\left(\frac{\varphi}{\delta}\right) \frac{-\nabla_{\mu}}{\left(\nabla_{\mathrm{ad}}-\nabla\right)} \text { for } \nabla_{\mu}<0
$$

where $\nabla_{\mu}$ is molecular weight gradient, $K$ denotes the thermal conductivity, $\mathrm{C}_{\mathrm{th}}=\frac{8}{3} \pi^{2} \alpha^{2}$ with $\alpha$ a free parame- ter, $\varphi=(\partial \ln \rho / \partial \ln \mu)_{\mathrm{P}, \mathrm{T}}$, and $\delta=-(\partial \ln \rho / \partial \ln \mathrm{T})_{\mathrm{P}, \mu}$. Evolutionary calculations show that thermohaline mixing extends between the outer wing of the H-burning shell and the inner boundary of the convective envelope, merging with the outer convection in a short time $(\sim 30 \mathrm{Myr}$ for a model with mass of the order of $1 \mathrm{M}_{\odot}$ ). Therefore, depending on the mixing efficiency, a significant amount of nuclear processed matter in the hotter layers of the H-burning shell can be dredged-up to the surface; an occurrence that allows to reproduce spectroscopic data.

This notwithstanding, there are still several shortcomings in the inclusion of this physical process in stellar model computations. At first, as it has been shown by [51], values of $\mathrm{C}_{\mathrm{th}} \sim 1000$ are required to reproduce the ${ }^{12} \mathrm{C} /{ }^{13} \mathrm{C}$ abundance pattern in halo RGB stars, but in order to reproduce the same trend for ${ }^{7} \mathrm{Li}$ abundances a quite smaller value is necessary $\left(\mathrm{C}_{\mathrm{th}} \sim 200\right)$. In addition, hydrodynamical simulations predict a different expression for the diffusion coefficient, that is reasonably reproduced by Eq. 1 with $\mathrm{C}_{\text {th }} \sim 10$ ([54]), i.e. the expected efficiency is much lower than needed to reproduce RGB spectroscopic data. In addition, recently [55] have provided compelling results showing that the surface chemical abundance predictions of stellar models accounting for thermohaline mixing strongly depend on the numerical assumptions on the mesh spacing and time resolution adopted in the evolutionary computations. As a consequence of these results, model predictions about surface chemical abundances should be treated with caution until a more firm assessment on how to manage with thermohaline mixing in stellar model computations is not achieved.

For long time, rotation and the associated rotational mixings have not - in general - been an ingredient of standard stellar models. This occurrence can be attributed largely to the increase in complexity and uncertainty read free parameters - of models that include rotation, and some estimates that rotation is expected to be at most a (small) perturbation to the structure of low-mass stars. A number of observational data, however, requires mixing mechanisms not present in standard stellar models, and mixing driven by rotation is at least a potential candidate. In addition, nowadays asteroseismic observations are clearly revealing the role played by rotation in the interiors of stars other than the Sun. From the point of view of the inclusion of rotation in model computations, but the huge increase in the complexity of the numerical equations that have to be solved, the main problems are related to our poor knowledge of the interaction between convection and meridional currents, of the efficiency of angular momentum transport and angular momentum loss during the core and shell H-burning stages. The inclusion of these processes in an evolutionary code requires the calibration of several free parameters, that, until a few years, were poorly constrained by standard observations.

In this context, a big improvement has been obtained - and more can be expected in the near future - thanks to seismic observations collected with Kepler satellite which allowed to measure the core rotation rates for hundreds of red giants $([56,57])$. The preliminary comparison between 
these empirical data and existing sets of models (see [58]) has shown that current RGB models underestimate the angular momentum extraction from the stellar core, i.e. the amount of torque between the core and the envelope seems to be significantly underestimated.

In order to increase the amount of angular momentum losses from the core, some possible 'candidates' have been identified such as internal gravity waves and large scale magnetic fields. However, a lot of work has to be done before we achieve a firm understanding of the role played by these individual physical processes.

\section{On the modelling of Red Clump stars}

Concerning the models for RC stars, the main sources of uncertainty are related to the estimation of the rate for the nuclear reaction ${ }^{12} \mathrm{C}(\alpha, \gamma){ }^{16} \mathrm{O}$, and of the treatment of convective core boundary.

The ${ }^{12} \mathrm{C}(\alpha, \gamma){ }^{16} \mathrm{O}$ reaction is one of the key reactions in stellar evolution. Its rate affects the $\mathrm{C} / \mathrm{O}$ ratio in the stellar core at the end of the central He-burning stage and, as a consequence, WD cooling times. In addition, during the central He-burning phase, when the abundance of $\mathrm{He}$ in the convective core is significantly reduced, $\alpha$-captures on carbon nuclei becomes strongly competitive with the triple- $\alpha$ reactions in terms of contribution to the nuclear energy budget, with the consequence that the cross-section for this reaction has a strong influence on the core $\mathrm{He}$ burning phase lifetime.

It is known that this reaction has a resonance and a very low cross-section $\left(\sim 10^{-17}\right.$ barn $)$ at low energies, and the nuclear parameters are difficult to measure experimentally or to predict from theory. In the last decade, the accuracy of this reaction rate has been significantly improved; however its estimate should be still affected by $\mathrm{a} \approx 30 \%$ uncertainty ([35]).

As discussed by [59], the treatment of convective boundaries during central helium burning is still an open question in stellar evolution calculations, and is handled in different ways by different stellar evolution codes. Recent advances in asteroseismic observations and techniques $([59,60])$ are starting to add very direct observational constraints to the core mixing process during the central He-burning phase, that coupled to theoretical inferences and indications from star counts in GGCs, make a strong case for the core mixed region to be extended beyond the Schwarzschild border.

Questions exist however about the treatment of this mixing beyond the canonical convective border: it is not so clear if a 'standard' overshoot scheme should be preferred to a numerical approach favouriting the formation of an extended, partially mixed region - 'semiconvective' zone - around the canonical convective core. For a detailed discussion on this issue we refer to $[35,60]$.

The uncertainty in the mixing treatment worsens as the core He-burning phase progresses. If no specific approach is used, the phenomenon of the so-called 'breathing pulses' occurs, that corresponds to a recursive phase of expansion of the convective core occurring when the $\mathrm{He}$ abundance in the core becomes quite low, and the feedback from the energy released by the ${ }^{12} \mathrm{C}(\alpha, \gamma){ }^{16} \mathrm{O}$ nuclear reaction dominates with respect the triple $-\alpha$ reactions (due to the paucity of He nuclei in the core).

The occurrence of the breathing pulses in real stars has been severely challenged by observations - mainly based on star counts in GGCs -, and their occurrence in models is attributed to the approximation of instantaneous mixing in the convective zones adopted in the model computations. Different methodological approaches have been designed to inhibit the breathing pulses, but they lead to different predictions for the following evolutionary phases (see [35], and references therein).

\section{Final remarks}

Huge improvements have been made regarding the major physics ingredients entering in stellar models 'cooking' such as opacities, nuclear reaction rates, equation of state, etc. Although - as briefly discussed above - there are still some issues - as the uncertain rates of some specific nuclear reactions - a realistic description of the main input physics needed for stellar computations seems within reach. On the other hand, we still face long-standing problems related to the treatment of mixing in both the stellar interiors and atmosphere. Despite the large efforts devoted to address this problem, we still need to use approximate numerical approaches with some (tunable) free parameter(s). In this context, the role played by rotation and rotationally-induced mixings also has to be fully investigated taking into account the updated constraints coming from seismic measurements on large samples of red giant stars.

We expect that quite important advances towards developing of a comprehensive stellar evolution theory, as well as more realistic stellar models, may come from constraints obtained by current (and future) asteroseismic surveys.

\section{References}

[1] A. Miglio, J. Montalbán, F. Baudin, P. Eggenberger, A. Noels, S. Hekker, J. De Ridder, W. Weiss, A. Baglin, A\&A 503, L21 (2009)

[2] L. Casagrande, V. Silva Aguirre, D. Stello, D. Huber, A.M. Serenelli, S. Cassisi, A. Dotter, A.P. Milone, S. Hodgkin, A.F. Marino et al., ApJ 787, 110 (2014)

[3] L. Casagrande, V. Silva Aguirre, K.J. Schlesinger, D. Stello, D. Huber, A.M. Serenelli, R. Schönrich, S. Cassisi, A. Pietrinferni, S. Hodgkin et al., MNRAS 455, 987 (2016)

[4] D. Sugimoto, M.Y. Fujimoto, ApJ 538, 837 (2000)

[5] A. Formicola, G. Imbriani, H. Costantini, C. Angulo, D. Bemmerer, R. Bonetti, C. Broggini, P. Corvisiero, J. Cruz, P. Descouvemont et al., Physics Letters B 591, 61 (2004)

[6] A. Weiss, A. Serenelli, A. Kitsikis, H. Schlattl, J. Christensen-Dalsgaard, A\&A 441, 1129 (2005) 
[7] A. Pietrinferni, S. Cassisi, M. Salaris, A\&A 522, A76 (2010)

[8] A. Pietrinferni, S. Cassisi, M. Salaris, F. Castelli, ApJ 612, 168 (2004)

[9] V. Silva Aguirre, S. Basu, I.M. Brandão, J. Christensen-Dalsgaard, S. Deheuvels, G. Doğan, T.S. Metcalfe, A.M. Serenelli, J. Ballot, W.J. Chaplin et al., ApJ 769, 141 (2013)

[10] T. Appourchaux, H.M. Antia, W. Ball, O. Creevey, Y. Lebreton, K. Verma, S. Vorontsov, T.L. Campante, G.R. Davies, P. Gaulme et al., A\&A 582, A25 (2015)

[11] F.J. Rogers, A. Nayfonov, ApJ 576, 1064 (2002)

[12] D.A. VandenBerg, F.J. Swenson, F.J. Rogers, C.A. Iglesias, D.R. Alexander, ApJ 532, 430 (2000)

[13] A. Dotter, B. Chaboyer, D. Jevremović, E. Baron, J.W. Ferguson, A. Sarajedini, J. Anderson, AJ 134, 376 (2007)

[14] O. Straniero, A\&AS 76, 157 (1988)

[15] M. Salaris, A. Chieffi, O. Straniero, ApJ 414, 580 (1993)

[16] J.W. Ferguson, D.R. Alexander, F. Allard, T. Barman, J.G. Bodnarik, P.H. Hauschildt, A. HeffnerWong, A. Tamanai, ApJ 623, 585 (2005)

[17] C. Neuforge, A\&A 274, 818 (1993)

[18] R.L. Kurucz, Atomic and Diatomic Molecular Opacities for Atmospheres and Envelopes, in American Astr. Soc. Meet. Abs. \#180 (1992), Vol. 24 of Bulletin of the American Astronomical Society, p. 779

[19] D.A. VandenBerg, B. Edvardsson, K. Eriksson, B. Gustafsson, ApJ 675, 746-763 (2008)

[20] K.S. Krishna Swamy, ApJ 145, 174 (1966)

[21] E. Böhm-Vitense, Zeitschrift fur Astrophysik 46, 108 (1958)

[22] M. Salaris, S. Cassisi, A\&A 487, 1075 (2008)

[23] M. Salaris, S. Cassisi, A. Weiss, PASP 114, 375 (2002)

[24] L. Deng, D.R. Xiong, K.L. Chan, ApJ 643, 426 (2006)

[25] V.M. Canuto, I. Mazzitelli, ApJ 389, 724 (1992)

[26] S. Pasetto, C. Chiosi, M. Cropper, E.K. Grebel, MNRAS 445, 3592 (2014)

[27] S. Pasetto, C. Chiosi, E. Chiosi, M. Cropper, A. Weiss, MNRAS 459, 3182 (2016)

[28] M.M. Miller Bertolami, M. Viallet, V. Prat, W. Barsukow, A. Weiss, MNRAS 457, 4441 (2016)

[29] A. Nordlund, R.F. Stein, M. Asplund, Living Reviews in Solar Physics 6, 2 (2009)

[30] R. Trampedach, M. Asplund, R. Collet, A. Nordlund, R.F. Stein, ApJ 769, 18 (2013)

[31] R. Trampedach, R.F. Stein, J. ChristensenDalsgaard, A. Nordlund, M. Asplund, MNRAS 445, 4366 (2014)

[32] R. Trampedach, R.F. Stein, J. ChristensenDalsgaard, ̊. Nordlund, M. Asplund, MNRAS 442, 805 (2014)

[33] M. Salaris, S. Cassisi, A\&A 577, A60 (2015)
[34] J.E. Vernazza, E.H. Avrett, R. Loeser, ApJS 45, 635 (1981)

[35] S. Cassisi, M. Salaris, Old Stellar Populations: How to Study the Fossil Record of Galaxy Formation (2013)

[36] S. Cassisi, M. Salaris, MNRAS 285, 593 (1997)

[37] M. Riello, S. Cassisi, G. Piotto, A. Recio-Blanco, F. De Angeli, M. Salaris, A. Pietrinferni, G. Bono, M. Zoccali, A\&A 410, 553 (2003)

[38] F. Fusi Pecci, F.R. Ferraro, D.A. Crocker, R.T. Rood, R. Buonanno, A\&A 238, 95 (1990)

[39] A. Di Cecco, G. Bono, P.B. Stetson, A. Pietrinferni, R. Becucci, S. Cassisi, S. Degl'Innocenti, G. Iannicola, P.G. Prada Moroni, R. Buonanno et al., ApJ 712, 527 (2010)

[40] S. Cassisi, A. Marín-Franch, M. Salaris, A. Aparicio, M. Monelli, A. Pietrinferni, A\&A 527, A59 (2011)

[41] M.S. Cunha, D. Stello, P.P. Avelino, J. ChristensenDalsgaard, R.H.D. Townsend, ApJ 805, 127 (2015)

[42] S. Cassisi, M. Salaris, G. Bono, ApJ 565, 1231 (2002), astro-ph/0110247

[43] D. Reimers, Memoires of the Societe Royale des Sciences de Liege 8, 369 (1975)

[44] M. Vieytes, P. Mauas, C. Cacciari, L. Origlia, E. Pancino, A\&A 526, A4 (2011)

[45] L. Origlia, F.R. Ferraro, S. Fabbri, F. Fusi Pecci, E. Dalessandro, R.M. Rich, E. Valenti, A\&A 564, A136 (2014)

[46] J. Heyl, H.B. Richer, E. Antolini, R. Goldsbury, J. Kalirai, J. Parada, P.E. Tremblay, ApJ 804, 53 (2015)

[47] J. Heyl, J. Kalirai, H.B. Richer, P. Marigo, E. Antolini, R. Goldsbury, J. Parada, ApJ 810, 127 (2015)

[48] M. Salaris, S. Cassisi, A. Pietrinferni, A\&A 590, A64 (2016)

[49] A. Miglio, K. Brogaard, D. Stello, W.J. Chaplin, F. D’Antona, J. Montalbán, S. Basu, A. Bressan, F. Grundahl, M. Pinsonneault et al., MNRAS 419, 2077 (2012)

[50] T.R. Bedding, B. Mosser, D. Huber, J. Montalbán, P. Beck, J. Christensen-Dalsgaard, Y.P. Elsworth, R.A. García, A. Miglio, D. Stello et al., Nature 471, 608 (2011)

[51] G.C. Angelou, V. D’Orazi, T.N. Constantino, R.P. Church, R.J. Stancliffe, J.C. Lattanzio, MNRAS 450, 2423 (2015)

[52] C. Charbonnel, J.P. Zahn, A\&A 467, L15 (2007)

[53] R. Kippenhahn, G. Ruschenplatt, H.C. Thomas, A\&A 91, 175 (1980)

[54] A. Traxler, P. Garaud, S. Stellmach, ApJL 728, L29 (2011)

[55] J.C. Lattanzio, L. Siess, R.P. Church, G. Angelou, R.J. Stancliffe, C.L. Doherty, T. Stephen, S.W. Campbell, MNRAS 446, 2673 (2015)

[56] B. Mosser, M.J. Goupil, K. Belkacem, J.P. Marques, P.G. Beck, S. Bloemen, J. De Ridder, C. Barban, S. Deheuvels, Y. Elsworth et al., A\&A 548, A10 
(2012)

[57] S. Deheuvels, G. Doğan, M.J. Goupil, T. Appourchaux, O. Benomar, H. Bruntt, T.L. Campante, L. Casagrande, T. Ceillier, G.R. Davies et al., A\&A 564, A27 (2014)

[58] M. Cantiello, C. Mankovich, L. Bildsten, J. Christensen-Dalsgaard, B. Paxton, ApJ 788,
93 (2014)

[59] T. Constantino, S.W. Campbell, J. ChristensenDalsgaard, J.C. Lattanzio, D. Stello, MNRAS 452, 123 (2015)

[60] D. Bossini, A. Miglio, M. Salaris, A. Pietrinferni, J. Montalbán, A. Bressan, A. Noels, S. Cassisi, L. Girardi, P. Marigo, MNRAS 453, 2290 (2015) 